\title{
Wer hat das Sagen im Gesundheitswesen?
}

\author{
Erwin Carigiet \\ Dr. iur., Spitaldirektor Stadtspital Triemli Zürich, Präsident der Kongresskommission
}

\begin{abstract}
Das Schweizer Gesundheitswesen ist ein komplexes System mit zahlreichen Akteurinnen und Akteuren, die es direkt oder indirekt beeinflussen. Dabei zeigt sich immer häufiger, dass das Einzelinteresse vor das Gesamtinteresse tritt.
\end{abstract}

\begin{abstract}
Am H+ Kongress am 11. November 2015 befassen wir uns mit dem Thema «Das Gesundheitswesen: Die Machtfrage» und versuchen dabei zu ergründen, wie die führenden Kräfte derzeit im Gesundheitswesen aufeinander wirken. Viele Beispiele aus jüngster Zeit zeigen, dass sich der Machtkampf im Gesundheitswesen in den letzten Jahren zugespitzt hat. So machte der Bundesrat letztes Jahr erstmals von seiner subsidiären Kompetenz Gebrauch und griff aktiv in den ambulanten Tarif TARMED ein, weil sich die Tarifpartner nicht einigen konnten. Dabei sprach er den Hausärztinnen und Hausärzten 200 Millionen Franken zu, wobei die Spitäler und Kliniken diese Zuschläge nicht abrechnen dürfen. Auf die anschliessende gemeinsame Beschwerde trat das Bundesverwaltungsgericht aus formalen Gründen nicht ein.
\end{abstract}

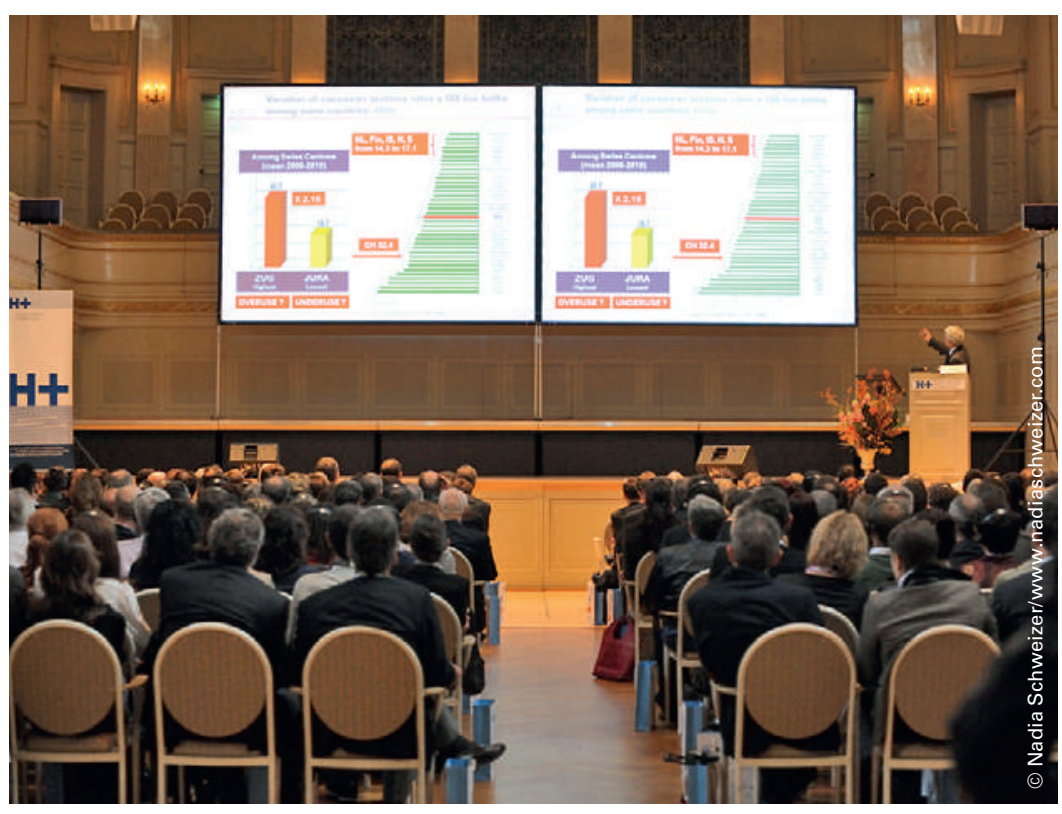

Jeder $\mathrm{H}+$ Kongress widmet sich einem speziellen Thema. Dieses Jahr lautet es: «Das Gesundheitswesen: Die Machtfrage».

\section{Gerichte als Schiedsrichter}

Der Fall TARMED ist nicht der einzige mit rechtlichem Ausgang. Es ist eine generelle Tendenz in den letzten Jahren, dass sich die Tarifpartner nicht mehr einigen können und deshalb den Gerichtsweg einschlagen. Das Bundesverwaltungsgericht befasst sich darum immer mehr mit Fällen rund um die Kostenermittlung und Tarif-Bildung unter SwissDRG. Die NZZ am Sonntag machte am 10. Mai 2015 einen weiteren Fall publik: santésuisse verklagt die vier Tarifpartner curafutura, FMH, H+ und MTK, um damit gerichtlich die Gründung der neuen TARMED Suisse AG zu verhindern. Ziel der neuen Organisation wäre es, den veralteten Tarif für ambulante Leistungen TARMED umfassend zu revidieren.

\section{Kantonale Einflussnahme}

Auch die Mehrfachrolle der Kantone als Eigner von Spitälern, Regulatoren und Genehmigungsbehörden ist regelmässig Gegenstand der öffentlichen Diskussion. Denn je nach Rolle ist auch die Haltung in Sachfragen unterschiedlich, was zu Interessenkonflikten führt. Um dem - zumindest teilweise - entgegenzuwirken, haben z.B. die Kantone Baselland und Sankt Gallen beschlossen, dass Regierungsräte nicht mehr in den Verwaltungsräten der Spitäler und Kliniken vertreten sein dürfen.

Im Kanton Bern ist die Spitalliste Gegenstand hitziger Diskussionen. Private Spitäler beklagen sich, dass der Sanitätsdirektor «seine» öffentlichen Spitäler bewusst besserstelle.

\section{Spitalmanagement auf dem Schleudersitz}

Ein bisher nur aus der Industrie bekanntes Phänomen zeigt sich vermehrt auch im Spitalwesen: Erfüllen Ver- 


\section{$\mathrm{H}+$ Kongress in Kürze}

$\mathrm{H}+$ organisiert alle zwei Jahre den $\mathrm{H}+$ Kongress für die wichtigsten Schweizer Akteure der Spitalbranche und des Gesundheitswesens im Allgemeinen.

Im Zentrum des $\mathrm{H}+$ Kongresses steht immer ein aktuelles Thema der Branche, das von verschiedenen Seiten beleuchtet wird. Der Anlass ist ausgerichtet auf Kadermitarbeitende der Ärzteschaft, der Pflege und des Managements sowie nationale Entscheidungsträger im Spital- und Gesundheitswesen, steht aber grundsätzlich allen Interessierten offen. Der Kongress am

waltungsräte oder das Spitalmanagement die Erwartungen seiner Trägerschaft nicht, werden Kündigungen ausgesprochen. So entliess der Regierungsrat im Kanton Baselland sowohl den CEO als auch den Verwaltungsratspräsidenten des Kantonsspitals Baselland, weil aufgrund der neuen strategischen Ausrichtung - eine grosse Anzahl von Ärztinnen und Ärzten mit der

\section{Es ist eine generelle Tendenz in den letzten Jahren, dass sich die Tarifpartner nicht mehr einigen können.}

Kündigung gedroht und diese Drohung zum Teil auch wahrgemacht hat. Im Spital Wallis trat der Verwaltungsratspräsident unter Protest zurück und das ganze Management wurde ausgewechselt. Der Kanton hat eine Untersuchung angeordnet. Der Schlussbericht dieser Untersuchung monierte Führungsmängel auf allen Ebenen.

\section{Einfluss des Volkes}

Die letztjährige Meinungsumfrage bei der stimmberechtigten Bevölkerung, die gfs.bern für $\mathrm{H}+$ durchführte, zeigte, dass die Befragten mit dem Schweizer Gesundheitssystem äusserst zufrieden sind und keine Reformen wollen. Entsprechend wurden alle grossen Reformen - mit Ausnahme des Gegenvorschlags zur Hausarztmedizin-Initiative - abgelehnt. Zudem löst es auch grosse Gegenwehr aus, wenn Spitäler oder Kantone verkünden, Spitalstandorte zu schliessen oder das Angebot eines Spitalstandorts einzuschränken. Dies spürten einige Regierungsrätinnen und Regierungsräte, die aufgrund einer solchen Ankündigung die Wiederwahl nicht erreichten.
11. November 2015 im Kulturcasino in Bern steht unter dem Motto: "Das Gesundheitswesen: Die Machtfrage». Ziel ist es, spannende Diskussionen unter den verschiedenen Berufsgruppen und Stakeholdern anzustossen. Neben dem Kongressplenum, für das eine Simultanübersetzung in Deutsch und Französisch gewährleistet ist, können die Besucher an zwei Workshops teilnehmen, wobei fünf zur Auswahl stehen.

Programm, weitere Informationen und Anmeldung $\mathrm{H}+\mathrm{Kon}-$ gress: www.hplus-kongress.ch

Unzufriedene Patientinnen und Patienten nehmen zudem über öffentliche wie auch soziale Medien Einfluss, indem sie ihrer Reklamation über einen Arzt, eine Ärztin oder ein Spital Gehör verschaffen.

\section{Machtdiskussion unter den Berufsgruppen}

Auch in den Spitälern und Kliniken selbst stellt sich immer wieder die Frage, wer die Führung bei der Festlegung der finanziellen Rahmenbedingungen bei der Behandlung der Patientinnen und Patienten übernimmt. Sind es die Pflegenden, die Ärztinnen und Ärzte oder doch das Management? Die Pflegeverbände haben sich beispielsweise stark gemacht, dass sie pflegerische Leistungen selbständig abrechnen dürfen und nicht nur nach ärztlicher Verordnung. Das Anliegen wird derzeit im Parlament behandelt.

\section{Strategien und Lösungsansätze gemeinsam diskutieren}

Die Diskussion zwischen den Berufsgruppen und Stakeholdern aktiv zu fördern ist eines der Ziele des H+ Kongresses 2015 und folgt dabei den Worten von Konrad Adenauer: "Macht und Verantwortung sind untrennbar verbunden.» Referentinnen und Referenten aus Forschung, Politik und Praxis werden am 11. November 2015 die aktuelle Situation analysieren und mögliche Lösungsansätze aufzeigen. In Workshops werden jeweils unterschiedliche Facetten der Machtfrage gemeinsam mit den Teilnehmenden beleuchtet. Die Workshops haben zum Ziel, Ideen für mögliche Lösungen zu entwickeln und kontrovers zu diskutieren. Wir freuen uns auf den anregenden und erfolgreichen Austausch! 